Cinémas

Revue d'études cinématographiques

Journal of Film Studies

\title{
Le mécanoïde et l'androïde : deux faces du mythe futuriste dans le cinéma d'avant-garde des années vingt
}

\section{Wanda Strauven}

Volume 12, numéro 3, printemps 2002

Cinélekta 4

URI : https://id.erudit.org/iderudit/000734ar

DOI : https://doi.org/10.7202/000734ar

Aller au sommaire du numéro

Éditeur(s)

Cinémas

ISSN

1181-6945 (imprimé)

1705-6500 (numérique)

Découvrir la revue

Citer cet article

Strauven, W. (2002). Le mécanoïde et l'androïde : deux faces du mythe futuriste dans le cinéma d'avant-garde des années vingt. Cinémas, 12(3), 33-51.

https://doi.org/10.7202/000734ar
Résumé de l'article

Cet article examine la mise en image filmique de l'adoration futuriste de la machine, en distinguant deux tendances : d'une part, la machine en tant que machine ou création non humaine qui peut être chargée de sens symbolique (de l'érotisme dans Impatience de Charles Dekeukeleire à la révolution prolétarienne dans La Grève de S. M. Eisenstein) et, de l'autre, la machine en tant que nouvelle créature aux formes humaines, qui très vite deviendra une menace pour son propre créateur. 


\section{Le mécanoïde et l'androïde: deux faces du mythe futuriste dans le cinéma d'avant-garde des années vingt}

\section{Wanda Strauven}

\section{RÉSUMÉ}

Cet article examine la mise en image filmique de l'adoration futuriste de la machine, en distinguant deux tendances: d'une part, la machine en tant que machine ou création non humaine qui peut être chargée de sens symbolique (de l'érotisme dans Impatience de Charles D ekeukeleire à la révolution prolétarienne dans $\mathrm{L} a$ Grève de S. M. Eisenstein) et, de l'autre, la machine en tant que nouvelle créature aux formes humaines, qui très vite deviendra une menace pour son propre créateur.

\section{ABST RACT}

This article examines the ways in which the Futurist adoration of the machine found expression in the cinema. Two tendencies are distinguished: first, that of the machine as machine, as a non-human invention that can become charged with symbolic meaning (from the eroticism of Charles D ekeukeleire's Impatience to the proletarian revolution in S.M. Eisenstein's Strike); and second, that of the new machine as a new being with a human form which quickly becomes a threat to its creator.

\section{Prolégomènes à un nouveau mythe}

Le futurisme italien, fondé en 1909 par Filippo Tommaso $M$ arinetti, prend d'emblée l'allure d'une véritable religion inspirée 
par le progrès technologique et la mécanisation de la société. L'enthousiasme effréné et catalytique des jeunes artistes futuristes face aux nouvelles conditions de vie s'explique par le caractère subit et rapide de l'évolution de l'économie italienne - à la différence de celle d'autres pays européens - qui, essentiellement agricole jusqu'en 1909-1910, est bouleversée par une industrialisation accélérée et prépondérante au moment même où naît le futurisme ${ }^{1}$. Avec cette métamorphose soudaine de la réalité italienne, des émotions neuves s'éveillent: «N ous, les futuristes, nous sommes les seuls primitifs d'une nouvelle sensibilité entièrement transformée», affirme le peintre U mberto Boccioni $(1975$, p. 60). Dans cet esprit, la production industrielle de l'automobile est d'un intérêt capital. La voiture - véhicule autonome et individuel - devient le symbole de l'élite et un des principaux fétiches futuristes. $N$ on seulement elle permet aux artistes (sous-entendu: cossus) d'expérimenter des sensations insolites, mais elle établit aussi un contact physique avec un corps mécanique, thème inspirateur d'une nouvelle poésie. Le même rôle révélateur sera dévolu, par la suite, à l'aéroplane, comme l'explique $M$ arinetti dans l'introduction du «M anifeste technique de la littérature futuriste» (1912) :

Ce fut en aéroplane, assis sur le cylindre à essence, le ventre chauffé par la tête de l'aviateur, que je sentis tout à coup l'inanité ridicule de la vieille syntaxe héritée de H omère (M arnetti, dans Lista, 1973, p. 133).

L'optimisme absolu devant la nouvelle civilisation se traduit dans l'art futuriste par l'exaltation de la beauté mécanique et par la valorisation de la vitesse, du dynamisme et du mouvement. L'objectif est de convaincre les autres, les soi-disant passéistes, des immenses possibilités qu'entraîne l'évolution technologique, d'accomplir - d'après les paroles de M arinetti (dans Lista, 1973, p. 142) - «le complet renouvellement de la sensibilité humaine sous l'action des grandes découvertes scientifiques». $D$ ans ce but, les futuristes prêchent une nouvelle foi, proclamant divines les machines et instaurant une mythologie technologique (et futurologique, c'est-à-dire convenant aux mondes futurs). En tant que porte-parole du mouvement, $M$ arinetti pro- 
fesse à plusieurs reprises cette doctrine blasphématoire qui se veut, sans équivoque, religieuse. Dès 1909, il fait prononcer à son personnage de $M$ afarka le futuriste, héros du roman éponyme, le discours suivant:

O ui, oui, mes frères, je vous ouvre les bras et je vous
embrasse sur mon cœur, car vous êtes dignes d'écouter
le verbe mystérieux de ma religion !... J J vous enseigne
à mépriser la mort, à vous nourrir de danger, à jouer
votre vie, ainsi que vous le faites, pour une idée, pour
un regard, pour un spectacle (M arinetti, 1910, p. 213)!

En 1916, M arinetti (dans Lista, 1973, p. 367) publie le manifeste "La nouvelle religion-morale de la vitesse», où il exhorte ses lecteurs à une liturgie sui generis («Si prier veut dire communier avec la divinité, courir à grande vitesse est bien une prière. Sainteté de la roue et des rails. II faut s'agenouiller sur les rails pour prier la divine vitesse»), visant en premier, comme nouveaux convertis, les sportifs ( «L'ivresse des grandes vitesses en auto est l'ivresse de se sentir fondu avec l'unique divinité. Les sportsmen sont les premiers catéchumènes de cette religion », p. 368).

Le culte futuriste est consacré à cette nouvelle idole qu'est la machine, à cette nouvelle muse des jeunes artistes, qui les initie au nouvel art mécanique. C'est ce même esprit qui continue à caractériser le soi-disant second futurisme des années vingt (la période héroïque du premier futurisme se situant entre 1909 et 1919), comme en témoignent de nombreux manifestes, entre autres, le «M anifeste de l'art mécanique futuriste» (1922) de Vinicio Paladini et Ivo Pannaggi, "L'esthétique mécanique» (1923) de Paladini et «L'idole mécanique» (1926) de Fillia, Curtoni et Caligaris. Le premier de la liste sera réédité en 1923, avec la collaboration d'Enrico Prampolini, dans une version augmentée, sous le titre "L'art mécanique»; il sagit d'un texte de base qui adapte et reformule clairement la défication de la machine:

La M achine donne aujourd'hui le rythme de la grande âme collective et des différents individus créateurs. La $M$ achine scande le chant du génie. La M achine est la 
nouvelle divinité qui éclaire, domine, distribue ses dons et punit, dans notre temps futuriste c'est-à-dire dévoué à la grande Religion du N ouveau (Paladini, Pannaggi et Prampolini, dans Lista, 1973, p. 223-224).

J'insiste sur cet aspect de divinisation (et, par conséquent, de mystification) afin de distinguer l'attitude des futuristes à l'égard de la machine de la banale attirance ou admiration pour la machine que l'on trouve déjà dans la littérature à une époque antérieure au futurisme ${ }^{2}$. Ébauché par M arinetti comme mouvement essentiellement littéraire et poétique, le futurisme se propage très vite dans toutes les disciplines artistiques - des arts plastiques à l'architecture, du spectacle (théâtre et cinéma) à la publicité, la mode et la décoration - et se constitue, de cette façon, non seulement un terrain vaste et solide pour la diffusion de sa nouvelle mythologie (qui est en partie, comme on verra plus avant, une réactualisation d'anciens mythes), mais encore une image plus systématique de l'imaginaire moderniste. $D$ ans un tel contexte, c'est avant tout la machine qui, en polarisant toutes les aspirations de la nouvelle société, sérige comme symbole privilégié et devient un thème/emblème mythique. Plus précisément, la machine est l'extériorisation, le symptôme maté riel de la transformation de la condition humaine auquel les futuristes ont voulu attribuer le statut de mythe.

\section{De la création du mythe futuriste à sa mise en image filmique}

La vénération futuriste pour la machine aboutit à l'attribution de propriétés humaines aux automates, à la croyance que chaque mécanisme a un esprit, une âme, voire un instinct. $D$ ans le «M anifeste technique de la littérature futuriste», $M$ arinetti (dans Lista, 1973, p. 135) proclame qu'il est temps de mettre fin à la contemplation anthropocentrique de la matière: c'est pourquoi il invite les poètes à «[r]emplacer la psychologie de I'homme, désormais épuisée, par l'obsession lyrique de la matière» et à «[a]usculter à travers les objets en liberté et les moteurs capricieux la respiration, la sensibilité et les instincts des métaux». D ans la conception marinettienne, la machine mène une vie autonome, indépendante des hommes. Ce nouvel être - Fortunato D epero (dans Lista, 1973, p. 201) parlera en 1914 
de «l'être vivant artificiel» - peut être conçu (ou modelé) de deux manières distinctes: soit la machine conserve son aspect d'objet mécanique, d'appareil, d'automate inhumain (c'est la machine en tant que machine, ce que j'appellerai le mécanoïde) ; soit elle revêt une forme reconnaissable, semblable à celle de I'homme (c'est l'apparition de l'androïde).

\section{- PREM IĖRE FACE : LE MÉCAN OÏDE}

La machine idolâtrée en tant que machine - en vertu de sa beauté extérieure (métallique, brillante) et de ses forces cachées, peu explorées - finit par remplacer l'homme et par s'imposer comme unique protagoniste dans le mythe futuriste. Tandis que le fondateur du futurisme exhorte à «[d]étruire le "Je" dans la littérature» (M arinetti, dans Lista, 1973, p. 135), Giacomo Balla étudie la course des automobiles et réalise maints tableaux d'analyse cinétique centrés sur le mouvement de la roue, tels que Automobile in corsa (Automobile en course, 1913), Velocità astratta (è passata l'automobile) (Vitesse abstraite (l'automobile est passée) , 1913), Espansione dinamica + velocità (Expansion dynamique + Vitesse, 1913), etc. Dans le manifeste «La splendeur géométrique et mécanique et la sensibilité numérique» (1914), $\mathrm{M}$ arinetti explique comment ses «sens futuristes» ont perçu pour la première fois la nouvelle beauté du monde mécanique. C'était sur le pont d'un cuirassé dreadnought:

La vitesse du navire, les distances des tirs fixées du haut de la passerelle dans la ventilation fraîche des probabilités guerrières, la vitalité étrange des ordres transmis par l'amiral et brusquement devenus autonomes et inhumains, à travers les caprices, les impatiences et les maladies de l'acier et du cuivre: tout cela rayonnait de splendeur géométrique et mécanique. Je perçus l'initiative lyrique de l'électricité courant à travers le blindage des tourelles quadruples, et descendant par des tubes blindés jusqu'aux soutes, pour tirer obus et gargousses jusqu'aux culasses, vers les volées émergeantes ( $M$ arinetti, dans Lista, 1973, p. 148).

La «sensation de plaisir, d'admiration, devant une belle machine» (Severini, dans Lista, 1973, p. 216) - sensation 
indéniable et inévitable selon le peintre Gino Severini - peut prendre des formes extrêmes allant du fétichisme à un véritable amour passionné et physique, dont témoigne le passage suivant du manifeste «L'art mécanique» de Paladini, Pannaggi et Prampolini (dans Lista, 1973, p. 223) :

\begin{abstract}
Les belles machines nous ont entourés, en se penchant amoureusement sur nous, et nous, sauvages instinctifs, découvreurs de tout mystère, nous nous laissons prendre dans leur ronde frénétique! Amoureux fous des machines, nous les avons possédées virilement, voluptueusement !
\end{abstract}

Le désir du contact charnel avec la machine est à la base d'un des films expérimentaux du réalisateur belge Charles D ekeukeleire: Impatience (1928). D ans ce drame à quatre personnages - la M ontagne, la M oto, la Femme et les Blocs abstraits - , le corps mécanique, celui de la M oto, est associé avec insistance au corps féminin, d'abord vêtu de cuir et ensuite nu. À travers le montage filmique, $D$ ekeukeleire démonte les deux corps dont les différents morceaux s'échangent entre eux. Le résultat est une sorte de symbiose moto-femme/femme-moto très suggestive, voire sensuelle, qui semble traduire cet «amour grandissant de la matière», ce "désir de la pénétrer et de connaître ses vibrations», que M arinetti (dans Lista, 1973, p. 150) évoque dans son manifeste «La splendeur géométrique et mécanique». Ces deux personnages, la $M$ oto et la Femme, entrent ensuite en interaction avec la M ontagne et les Blocs abstraits, comme si le réalisateur avait l'intention de mettre en scène - selon la suggestion de M arinetti (dans Lista, 1973, p. 250) - des «analogies profondes entre l'humanité, le monde animal, le monde végétal et le monde mécanique». $D$ ans la poétique marinettienne, l'analogie est une espèce de métaphore condensée, née d'associations irrationnelles. Comme l'explique le poète dans son «M anifeste technique de la littérature futuriste», I'analogie «n'est que l'amour immense qui rattache les choses distantes, apparemment différentes et hostiles» ( $M$ arinetti, dans Lista, 1973, p. 134). Par-dessus tout, il sagit de faire suivre immédiatement chaque substantif (sans locution conjonctive) par son 
«double», c'est-à-dire par le substantif «auquel il est lié par analogie». Cette formule littéraire est illustrée par $M$ arinetti (dans Lista, 1973, p. 133) dans les exemples suivants: «hommetorpilleur, femme-rade, foule-ressac, place-entonnoir, porterobinet». L'on pourrait ajouter les analogies «femme-moto» et «femme-montagne» d'Impatience.

Ballet mécanique (1924) de Fernand Léger représente une autre illustration filmique de l' «obsession lyrique de la matière», basée sur le même principe analogique. En introduisant des fragments du corps humain dans ce ballet d'objets et de mécanismes inhumains, Léger a voulu faire ressortir la concordance entre la machine et l'homme, ou plutôt la non-supériorité de I'humain par rapport à l'inhumain. Ballet mécanique est une application du «nouveau réalisme», esthétique dont Léger $(1926$, p. 7) explique le principe dans la revue new-yorkaise The Little Review :

A pipe - a chair - a hand - an eye - a typewriter
- a hat - a foot, etc., etc.
Let us consider these things for what they can contri-
bute to the screen just as they are - in isolation -
their value enhanced by every known means.
In this enumeration I have purposedly included parts
of the human body in order to emphasize the fact that
in the new realism the human being, the personality, is
very interesting only in these fragments and that these
fragments should not be considered of any more
importance than any of the other objects, listed.

La même importance est accordée à tous les éléments, humains et inhumains. Rappelons que Léger sinspira pour la réalisation de Ballet mécanique du chef-d'œuvre d'Abel Gance La Roue, plus particulièrement des séquences projetées à part au Salon d'automne en 1923 et au C.A.S.A. (Club des amis du septième art) en avril 1924. II s'agissait de passages isolés et sélectionnés en fonction de leur expressivité visuelle et rythmique, indépendante de la diégèse ${ }^{3}$. Ces extraits de pur mouvement mécanique, ces danses de roues, de rails, de pistons, etc. ont stimulé chez les jeunes avant-gardistes, tels Fernand Léger et René Clair, la réalisation de courts métrages non narratifs ${ }^{4}$. À 
cette même époque, Léger collabore au film de style Art déco de M arcel L'H erbier L'Inhumaine (1925), pour lequel il construit le laboratoire du «jeune ingénieur passionné de mécanique, de sport et de la féerie des sciences modernes ${ }^{5}$, nommé Einar N orsen. C'est dans cet atelier futuriste plein d'ingénieuses machines, que la cantatrice Claire Lescot sera ramenée à la vie (la technologie ressuscite l'homme). Du point de vue visuel, l'imaginaire mécanique de Léger est comparable à celui du scénographe futuriste Prampolini, plus particulièrement dans son projet de décor pour II Vulcano de M arinetti dans la mise en scène de Luigi Pirandello (au théâtre Valle, à Rome, en 1926). Le protagoniste de ce spectacle est la machine qui sert à contrôler les coulées de lave. Pour ce qui est des machines de l'ingénieur $\mathrm{N}$ orsen, celle qui revêt un intérêt majeur en termes futuristes est le système télévisuel expérimental qui lie l'atelier au monde extérieur. C ette invention permet de projeter sur un écran tous les auditeurs de Claire Lescot, dispersés dans le monde entier, et de produire ainsi un sens d'ubiquité, de simultanéité et de compénétration concepts centraux de la poétique marinettienne.

La problématique mécanique/machinique est traitée d'une façon très différente par le grand maître du montage, $S$. M. Eisenstein. L'ouverture de Stachka (La G rève, 1924) révèle nettement l'esprit constructiviste du réalisateur: le décor industriel - l'usine avec sa fonderie et son atelier de montage (!), la machinerie, les locomotives, l'amas de roues - constitue un cadre symbolique, chargé de sens, qui emporte les ouvriers dans leur révolte. La machine est conçue par Eisenstein comme une métaphore de la révolution prolétarienne, de la liberté potentielle de l'homme. $D$ ans plusieurs plans, il opère une véritable fusion de l'homme et de la machine; retenons à ce propos l'image des trois ouvriers, les bras croisés, avec en surimpression une grande roue en mouvement. L'analogie «homme-machine» revient dans Bronenosec Potemkin (Le Cuirassé Potemkin, 1925), plus précisément à travers un short-cutting d'images métonymiques des deux identités divergentes: mains-roues, visagemanomètre, poitrine-chaudière ${ }^{6} \ldots$ M oyennant ces minianalogies, Eisenstein fond ensemble l'humain et l'inhumain, le spirituel et le mécanique. 
D ans les années vingt, la représentation de la machinerie et du paysage modernistes atteindra son apogée dans les documentaires urbains, les soi-disant «symphonies de ville», où la métropole fonctionne en tant que lieu de rencontre des nouveaux protagonistes, les machines. Un certain fétichisme industriel - corrélatif à cette passion pour les «villes modernes et trépidantes ${ }^{7} \gg-$ caractérise ainsi Berlin die Symphonie einer Großstadt (Berlin, symphonie d'une grande ville, 1927) de Walter Ruttmann. La façon dont ce film met en valeur l'usine est très révélatrice: Ruttmann offre au spectateur une image poétique, stylisée de la machinerie, qui tient du véritable hommage futuriste à la «beauté mécanique» et semble répondre au onzième point du premier manifeste futuriste «Fondation et $M$ anifeste du Futurisme» (1909) :

\begin{abstract}
N ous chanterons les grandes foules agitées par le travail, le plaisir ou la révolte; les ressacs multicolores et polyphoniques des révolutions dans les capitales modernes; la vibration nocturne des arsenaux et des chantiers sous leurs violentes lunes électriques; [... ] les usines suspendues aux nuages par les ficelles de leurs fumées [... ] (M arinetti, dans Lista, 1973, p. 87).
\end{abstract}

Au demeurant, le film thématise exemplairement le mouvement et la vitesse avec des images chaotiques de la circulation et la mise en scène de courses de bicyclettes et d'automobiles (avec des plans isolés de la roue).

La «symphonie de ville» tournée à M oscou par D ziga Vertov, Chelovek s kinoapparatom (L'H omme à la caméra, 1929), peut être rapprochée de celle de Ruttmann : les deux documentaires racontent la journée entière d'une ville, de l'aube jusqu'à la nuit; dans leur totalité, ils constituent en quelque sorte une métaphore de la respiration d'une métropole. Tout en appliquant les principes des kinoki, Vertov reprend quelques thèmes traités par Ruttmann comme celui de la circulation chaotique (images en surimpression de carrefours envahis par des automobiles et des tramways) ou de l'usine (mise en scène progressivement accélérée du travail à la chaîne). $M$ ais, on le sait, l'intérêt est ailleurs: en poursuivant l'opérateur, en le filmant en pleine 
action, Vertov veut initier le spectateur à l'art du «ciné-eil », à ce nouveau mode de voir, de scruter le monde. Ainsi le vrai protagoniste n'est-il pas l'homme mais la caméra. Ce mécanoïde qui capte le monde avec un œil plus parfait que l'œil humain s'impose sans répit tout le long du film et finit par s'animer: le trépied commence à se mouvoir - comme «une grosse araignée ${ }^{8} \gg$ - et l'appareil cinématographique s'installe, de son propre mouvement, sur lui. Avec cette image de la caméra animée, dotée d'une vie (et surtout d'un regard), Vertov dépasse amplement la superficialité des futuristes italiens qui, dans leur enthousiasme, n'exploreront jamais à fond la véritable fonction de la machine (moins encore celle de l'appareil cinématographique) au sein de la vie émotionnelle de l'homme.

II semble donc que le mécanoïde soit traité dans le cinéma d'avant-garde des années vingt comme un acteur plutôt louable, chargé d'un sens positif (érotique, plastique, révolutionnaire ou autre). Cependant dans La Roue, la menace est déjà présente: la machine n'est pas un acteur inoffensif, elle peut se révéler meurtrière. Évoquons ici La Révolte des machines ou La Pensée déchaînée, scénario que Romain Rolland compose en 1921, en collaboration avec le dessinateur Frans $M$ asereel. Publié seulement après la mort de l'écrivain, ce scénario ne fut jamais porté à l'écran. II s'agissait de donner une image du mécanoïde en tant que monstre, être destructeur qui se révolte contre son créateur et cherche à supprimer la race humaine. Le génie de l'homme réussira toutefois à détruire les machines: en semant la discorde et en excitant la jalousie entre elles, il déclenche une véritable guerre des machines, qui finissent par s'entretuer. M ais I'homme se remettra à inventer, à dessiner de nouvelles machines et ainsi le cycle recommencera.

La description que Rolland donne des machines révèle un esprit assez inventif. II range les «M achines de formidable puissance» d'un côté, et les «M achines psychologiques» de l'autre. Parmi les premières réapparaît l'image de l'araignée:

L'une soulève une masse monstrueuse et la porte négligemment au-dessus de l'honorable assemblée. L'autre a cent bras d'acier qui se déroulent et se hérissent de toute part, comme une araignée gigantesque (Rolland, 1947, p. 54). 
Le deuxième groupe se réduit principalement à la machine «pour lire dans la pensée». II s'agit d'une invention bien curieuse qui permet de projeter, à la manière d'une lanterne magique, les pensées des gens:

\begin{abstract}
Elle a la forme d'un œil, au bout d'une trompe d'élé phant, qui sallonge, se pose par un bout sur le crâne du patient, et qui, par l'autre bout, comme une lanterne magique, projette sur l'écran ce qu'on voit dans le crâne: I'animal qui sommeille, les pensées secrètes gigantesques (Rolland, 1947, p. 55).
\end{abstract}

La révolte qui sannonce déjà vers la fin du Premier Acte éclate dans I'Acte II, quand les machines de guerre (batteuses, faucheuses, scies mécaniques, perforatrices, grues... ) commencent à détruire les villes et les champs. Cette vision catastrophique caractérise davantage la deuxième face du mythe de la machine.

\title{
- DEUXIÈM E FACE : L'AN DROÏDE
}

La tendance à concevoir la nouvelle divinité mécanique à l'image de l'homme se manifeste assez tôt dans l'esprit des futuristes et ne traduit, en fait, qu'un désir ancestral de pouvoir de création - topique récurrent dans la mythologie grecque. $\mathrm{De}$ cette mythologie émergent deux mythes: d'une part, le mythe de Prométhée, qui créa l'humanité en modelant de l'argile; et d'autre part, celui d'H éphaïstos, dieu du feu et des forges, célè bre pour ses inventions mécaniques pour ainsi dire cybernétiques ${ }^{9}$. Du mythe de Prométhée sont tirées des légendes du Golem et de Frankenstein, tandis que celui d'H éphaiistos constitue, en vertu de sa connaissance des machineries, l'archétype de la révolution industrielle. Comme l'on verra, I'androïde futuriste n'est pas un monstre fabriqué avec des dépouilles humaines ou du limon, mais un assemblage de métaux forgés, pourvu d'un cœur mécanique, infaillible et durable: le moteur.

Déjà dans sa pièce préfuturiste Poupées électriques (1909), qui sera reprise en pleine saison futuriste dans une version réduite au deuxième acte et intitulée Elettricità (puis Elettricità sessuale), $M$ arinetti introduit des personnages mécaniques d'aspect humain. Ces «poupées électriques», inventées et construites par 
l'ingénieur John Wilson (rebaptisé Riccardo M arinetti dans la synthèse futuriste), peuvent être mises en marche en vue d'imiter certaines actions humaines, telles que tousser ou ronfler, lire un journal ou faire de la dentelle. La présence de ces automates excite le couple Wilson/M arinetti dans leurs actes d'amour: c'est grâce à eux que l'électricité augmente dans l'air.

Toujours en 1909, M arinetti (1910, p. 277) conçoit Gazourmah, «H éros sans sommeil », qui est doté d'une «musculature géante» et de deux ailes immenses «déployées sur un treillis savant d'acier, de bambou et de nerfs d'hippopotame». II sagit du fils artificiel de $M$ afarka le futuriste, qui le construit lui-même à I'instar de D édale, autre prototype du génie technique emprunté à la mythologie grecque ${ }^{10}$. La naissance de cet homme mécanique, engendré sans l'aide d'une femme, symbolise la maîtrise totale de la matière et le pouvoir de la façonner librement. Ainsi l'explora tion et la domination des matériaux mènent à l'acte créateur, à cette tentation d'imiter Dieu. En 1912, M arinetti (dans Lista, 1973, p. 137) annonce dans le «M anifeste technique de la littérature futuriste» |'avènement du man-made man :

\begin{abstract}
Par l'intuition, nous romprons I'hostilité apparemment irréductible qui sépare notre chair humaine du métal des moteurs. Après le règne animal, voici le règne mécanique qui commence! Par la connaissance et l'amitié de la matière, dont les savants ne peuvent connaître que les réactions physico-chimiques, nous préparons la création de l'homme mécanique aux parties remplaçables.
\end{abstract}

Le concept d'un être artificiel «aux parties remplaçables» sous-entend non seulement l'idée du montage mécanique, mais il se rapproche aussi de l'opération créatrice effectuée par le «ciné-œil » vertovien. Tel qu'il résulte du «C onseil des Trois» (1923), le montage filmique est lié explicitement par Vertov (1972, p. 30) et ses adeptes à l'acte sublimé de la création:
M oi, ciné-œil, je crée un homme beaucoup plus parfait que celui qu'a créé Adam, je crée des milliers d'hom- mes différents d'après différents dessins et schémas préa- lables. Je suis le cinéœil. 
À l'un je prends les bras, plus forts et plus adroits, à l'autre je prends les jambes, mieux faites et plus véloces, au troisième la tête, plus belle et plus expressive, et, par le montage, je crée un homme nouveau, un homme parfait.

Quant au montage en termes mécaniques, je renvoie à la comédie utopiste R.U .R. (1920) de Karel Capek. D ans le prologue de cette pièce, qui lance le terme «robot » désignant I'homme artificiel et mécanique ${ }^{11}$, le directeur général des usines R.U.R. (Robots Universels de Rossum) accueille la jeune $\mathrm{H}$ élène Glory, à laquelle il explique les diverses étapes de la fabrication du robot:

\begin{abstract}
Ensuite, il y a l'atelier de montage où l'on met tout cela ensemble, vous comprenez, comme on monte des automobiles. Chaque ouvrier n'ajoute qu'une parcelle, cela court automatiquement à l'autre, puis au troisième et ainsi de suite à l'infini. C 'est ce qu'il y a de plus intéressant à voir (Čapek, 1924, p. 7).
\end{abstract}

Comme dans le scénario de Rolland, R.U.R. met en scène la prise du pouvoir par les machines (cette fois-ci des androïdes) et la destruction quasi totale de la race humaine: la pièce est l'expression du scepticisme civil de l'auteur et de son appréhension des conséquences négatives d'un progrès technique unilatéral. O bservons que le thème de l'angoisse industrielle se dessine aussi à l'intérieur même du futurisme, plus particulièrement dans la «synthèse tragique en trois temps» L'angoscia delle macchine (L'angoisse des machines), composée par le futuriste sicilien Ruggero Vasari en 1925. II s'agit d'une pièce de science-fiction qui se déroule comme un véritable récit apocalyptique. Q uand Tonkir, à la fois fondateur et l'un des trois despotes du Royaume des $M$ achines, commence à désespérer devant le spectacle de sa propre création, les deux autres despotes Bacal et Singar décident de le tuer. M aisTonkir se suicide et meurt, délirant, dans les bras d'une de ses créatures. Sa mort provoque immédiatement un dérèglement du système central : le règne croule, les «condamnés aux machines» se libèrent et la $M$ achine est victorieuse!

L'androïde de la scène futuriste, qui fera aussitôt son entrée dans le cinéma de science-fiction, revêt l'apparence d'un robot. 
S'il est vrai que déjà à la fin des années dix, Fortunato $D$ epero crée de véritables prototypes de robot, entre autres, dans la peinture Ballerina idolo (1917) et le fusain Automates (1917), c'est davantage la scénographie des années vingt qui déterminera l'aspect visuel de ce nouvel être artificiel. Songeons, par exemple, au costume robotique réalisé par Pannaggi pour Ballo M eccanico Futurista, spectacle qu'il élabore en 1922 avec la collaboration de Paladini et qui consiste en un dialogue entre un mannequin mécanique (robotique) et un fantoche humain. D epero, pour sa part, compose en 1923 le ballet mécanique Anihccam del 3000 pour lequel il dessine des locomotives anthropomorphes. Pour ce qui est des costumes des «condamnés aux machines» de la susdite pièce de Vasari, il y a d'une part les dessins de Pannaggi, qui conçoit des androïdes mécano-métalliques composés de formes cubiques et marqués d'un sigle numérique, et d'autre part ceux de Vera Idelson, élève d'Alexandra Exter, qui opte pour un aspect moins robotique et plus stylisé (ou abstrait ${ }^{12}$ ). D ans ce contexte, il faudrait aussi mentionner les costumes des $M$ artiens dessinés par Alexander Exter pour le film Aelita (1924) de Jakov Protazanov. Cette superproduction de science-fiction, accusée à l'époque par les critiques du Parti de "commercialisme», est imposante par l'épisode extra-terrestre tourné dans une mise en scène constructiviste et peuplé d'une armée de robots et d'une foule d'ouvriers esclaves. Ce sont surtout ces derniers qui avec leurs têtes (sans visage) de forme cubique présentent une analogie frappante avec l'œuvre des décorateurs futuristes.

Dès 1922, des robots d'apparence futuriste font leur entrée dans le cinéma avec L'U omo meccanico (L'H omme mécanique), film tout à fait remarquable réalisé en Italie, en 1921, par le comique français André $\mathrm{D}$ eed, alias $\mathrm{C}$ retinetti ${ }^{13}$. Le synopsis du film est le suivant : une bande commandée par la scélérate $M$ ado vole le projet de construction de robots et fabrique ainsi le premier homme mécanique au caractère plutôt destructeur. Le personnage D 'Arca forge un deuxième robot identique qui est mis en circulation pour combattre le premier. La bataille entre les deux robots, télécommandés ${ }^{14}$ respectivement par $M$ ado et D 'Arca, se termine dans une explosion spectaculaire due à un court-circuit qui a été occasionné par Saltarello (André D eed). 
Les costumes des robots ressemblent beaucoup aux dessins de $D$ epero et surtout aux personnages-locomotives de son ballet mécanique de 1923. Ceci pourrait être l'indice d'une influence dans le sens inverse, c'est-à-dire de l'image filmique sur l'imagerie futuriste ${ }^{15}$.

M etropolis (1926) de Fritz Lang reprend le thème čapekien des ouvriers au service des machines (ou celui de Vasari des "condamnés aux machines»). Cette épopée de sciencefiction, située en 2026, demeure spectaculaire aujourd'hui encore pour son architecture visionnaire (d'inspiration futuriste) et l'apparition de la femmerobot. Cette dernière incarne encore le mythe de l'androïde, cette fois-ci dans sa version féminine. Fabriquée par l'inventeur Rotwang qui rêve de ressusciter en elle un vieil amour, la robote est colorée d'un caractère fortement érotique. Le montage des parties métalliques qui constitue son corps fait ressortir une grâce féminine presque palpable. Et Rotwang représente en quelque sorte l'artiste moderne qui, conformément à l'ancien mythe de Pygmalion, tombe amoureux de sa propre création. Ensuite, il transformera sur l'ordre du M aître de $M$ etropolis cette femme métallique en une créature en chair et en 0 , identique à la jeune femme $M$ aria, qui, à cause de ses sermons prophétiques auprès des ouvriers, est considérée dange reuse par les technocrates. Sa copie est conçue dans le but de devenir une arme démagogique. La vraie et la fausse $M$ aria, interprétées toutes les deux par la même actrice Brigitte $\mathrm{H} \mathrm{elm}$, symbolisent la dualité de l'être féminin, personnifiant respectivement la sainte (pureté) et la prostituée (immoralité). En même temps, elles opposent l'humain au mécanique, ce qui provoquera une révolte chez les ouvriers ${ }^{16}$.

D ans M etropolis, l'androïde finit par se confondre avec I'homme: les ouvriers ne distinguent pas les deux femmes et c'est seulement sur le bûcher que la fausse $M$ aria reprend sa forme métallique de robot. L'impossibilité de différencier de l'extérieur le robot de l'homme - idée qui est déjà développée par Capek dans R.U.R. et qui sera reprise, entre autres, par Ridley Scott dans Blade Runner (1982) avec la race des Replicants - signifie en quelque sorte la fin, ou plutôt la négation, du mythe de la machine tel qu'il fut instauré par les futuristes: 
la beauté mécanique et la splendeur des métaux sont négligées en faveur d'un anthropomorphisme poussé à l'extrême ${ }^{17}$.

\section{Conclusion}

Par cette étude schématique du mythe futuriste et de sa mise en image filmique deux tendances se sont dessinées. D 'une part, la fascination pour les avancées mécaniques et industrielles semble susciter des créations expérimentales, inspirer des visions poétiques mettant en scène un nouvel acteur: la machine. Les artistes et les réalisateurs d'avant-garde sont charmés par la machine en tant que machine, pour ses valeurs intrinsèques, ses qualités spécifiques, c'est-à-dire non humaines. D ans les diffé rentes expériences filmiques, un des leitmotiv est la roue, qui en raison de sa forme et de son mouvement fonctionne comme métaphore de l'évolution (ou révolution) technologique. C ependant, le désir d'attribuer à la machine une forme humaine correspond à un autre besoin, soit de rendre ce nouveau protagoniste reconnaissable, de l'incarner pour qu'il ne reste pas une abstraction. La science-fiction intègre la machine au monde des hommes en tant que nouvelle créature (et non en tant que nouvelle création) dont la ressemblance avec l'homme implique une rivalité avec ce dernier, voire un sentiment d'hostilité à son égard. En fin de compte, c'est l'apparition de l'androïde qui commencera à ébranler et à assombrir l'utopie futuriste, à la transformer en une black utopia.

Université d'Amsterdam

\section{NOTES}

1. Voir à ce propos Reyner Banham (1960, p. 101): «[... ] the process of industrialisation in the north, which had begun with the introduction of steam power into textile factories in the 1860's was violently accelerated in the period of Futurism. Production of textiles trebled in the period between 1900 and 1912, the output of iron and steel rose from 300,000 metric tons to almost 1,000,000 metric tons in the same period, and other industries experienced comparable increases. At the same time, the creation of an automobile industry capable of producing machines that could hold their own in international competition gave industry a glow of psychological prestige that mere increase in quantity of established products could not have done».

2. Les précurseurs du futurisme sont en effet très nombreux; songeons, par exemple, aux fantaisies scientifiques de Jules Vernes et H. G. Wells, aux contes d'E.T.A. 
H offmann, à L'Ève future (1887) de Villiers de I'Isle Adam, au Surmâle (1902) d'Alfred Jarry, au D octeur Lerne (1908) de M aurice Renard, etc. D u point de vue théorique, l'essai de M ario M orasso La nuova arma. La macchina (1905) est d'un grand intérêt. Parmi ces écrits préfuturistes sur la fascination des machines, il faudrait aussi mentionner le drame en trois actes Poupées électriques, conçu par M arinetti en 190507 et publié à Paris, chez Sansot, en 1909. II s'agit d'une parodie de la sentimentalité et de la moralité bourgeoises dans laquelle des fantoches font fonction de stimuli sexuels. La pièce fut représentée en traduction intégrale italienne (avec le titre La donna è immobile) une seule fois, le soir du 15 janvier 1909, à Turin. Le deuxième acte de la pièce sera ensuite adopté par $M$ arinetti dans le cadre du théâtre synthétique futuriste (cf. infra).

3. D ans «Essai sur la valeur plastique du film d'Abel G ance La Roue», Léger (1922, p. 5) fait l'éloge de l'«état plastique» du film qui, sel on lui, «se localise presque exclusivement dans les trois premières parties, là où la machine joue un rôle prépondérant, là où la machine devient "personnage principal," acteur principal». Et Léger poursuit: "Ce sera l'honneur d'Abel Gance d'avoir imposé avec succès au public un "acteur objet". » Observons que dans La Roue, la présence de la machine ferroviaire reste obsédante jusqu'à la fin : la locomotive est filmée tout le long du film comme un vrai personnage de drame, comme un vrai mécanoïde, à la fois menaçant et touchant.

4. Pour plus de détails à ce sujet, je renvoie à Richard Abel (1986, p. 394-395).

5. II s'agit d'un des intertitres du film.

6. À propos de ce short-cutting analogique, voir Arnold Hauser (1962, p. 241): «men working desperately, engine-room of the cruiser; busy hands, revolving wheels; faces distorted with exertion, maximum pressure of the manometer; a chest soaked with perspiration, a glowing boiler; an arm, a wheel ; a wheel, an arm ; machine, man; machine, man ; machine, man. » Et $\mathrm{H}$ auser ajoute: «Two utterly different realities, a spiritual and a material, were joined together here, and not only joined but identified, in fact, the one proceeding from the other. »

7. Les «villes modernes et trépidantes» figurent parmi les «Lieux habités par le D ivin » dans le manifeste «La nouvelle religion-morale de la vitesse» ( $M$ arinetti, dans Lista, 1973, p. 368).

8. La métaphore de la caméra-araignée est utilisée péjorativement par Luigi Pirandello dans son roman Quaderni di Serafino Gubbio operatore (On tourne. Notes de Serafino Gubbio opérateur, 1925, p. 83), qui décrit d'une manière mordante le monde du cinéma et cet appareil monstrueux qu'est la caméra: «[Les acteurs] se sentent esclaves, eux aussi, de cette petite machine à la voix stridente, qui sur son trépied ressemble à une grosse araignée aux aguets, suçant, absorbant leur réalité vivante pour en faire une image évanescente, passagère, un jeu fait, pour le public, d'illusion mécanique. »

9. H éphaïstos fabriqua, entre autres, deux servantes en or, semblables à des vierges vivantes, et un géant de bronze nommé Talos. Pour les inventions mécaniques, cf. H omère, L'Iliade, chant XVIII , v. 375-380 (fabrication de vingt trépieds automatiques) ; v. 417-420 (apparition des deux servantes en or). Le mythe de Talos, homme d'airain, est présent dans Apollonios de Rhodes, Les Argonautiques, chant IV, v. 16331688.

10. G azourmah serait alors la réincarnation moderne d'Icare. Le refus des anciens mythes de la part des futuristes est, en effet, assez contradictoire. Pourquoi opposer à l'exaltation d'I care l'exaltation de I'héroïsme aéronautique? Voir à ce propos Roberto Tessari (1973, p. 220).

11. Le mot tchèque robota, signifiant «travail forcé», est utilisé par Čapek pour nommer les personnages artificiels, qui sont des ouvriers-esclaves de I'homme. 
12. Cf. Günter Berghaus (1998, p. 505).

13. Bien que L'U omo meccanico ait obtenu le visa de censure en novembre 1921, sa première romaine n'eut lieu qu'en octobre de l'année suivante. C $f$. Vittorio $M$ artinelli (1996, p. 349). Retrouvé en partie et restauré par la cinémathèque de Bologne, le film fut projeté opportunément lors d'une «soirée futuriste» organisée au centre Pompidou, à Paris, en janvier 1996.

14. Les robots sont commandés à distance à partir d'une chambre dans laquelle leurs mouvements et leurs actions sont projetés sur un écran (exemple précoce de métacinéma, de mise en abyme de l'écran, comparable au système télévisuel d'Einar $\mathrm{N}$ orsen dans L'Inhumaine).

15. Sur l'antériorité de l'image filmique par rapport à la scène futuriste, voir $M$ ichele Canosa (1991, p. 123-124). En plus d'exclure toute influence des premières mises en scène de la pièce R.U.R. sur le film de D eed, Canosa met en doute la possibilité que L'U omo meccanico ait inspiré directement le costume du mannequin mécanique du Ballo meccanico futurista, représenté à Rome le 2 juin 1922 (c'est-à-dire avant la première romaine du film). S'abstenant de définir L'U omo meccanico comme un film pré curseur de l'«esthétique mécanique», il constate simplement que l'avant-garde italienne trouve «un motif thématique figuratif (mécanographique) » dans le cinéma populaire.

16. À propos de cette dichotomie de la figure féminine, voir Guntram Geser (1996, p. 40-41).

17. L'ère de l'esthétique de l'androïde mécanique n'est cependant pas condamnée à disparaître: elle connaîtra plusieurs recrudescences, en particulier dans les superproductions américaines de sciencefiction. Ensuite, un nouveau mythe surgit: celui du cyborg, synthèse optimale de la machine et de l'homme. L'homme renonce à l'intégralité de son corps physique afin de l'améliorer par l'implantation de prothèses, d'organes mécaniques, tout en préservant son cerveau et son âme.

\section{RÉFÉREN CES BIBLIOGRAPHIQUES}

Abel, 1986: Richard Abel, French Cinema: the First Wave, 1915-1929, Princeton, Princeton University Press, 1986.

Banham, 1960: Reyner Banham, Theory and D esign in the First M achine Age, London, The Architectural Press, 1960.

Berghaus, 1998 : Günter Berghaus, Italian Futurist T heatre, 1909-1944, O xford, Clarendon Press, 1998.

Boccioni, 1975 : U mberto Boccioni, D ynamisme plastique. Peinture et sculpture futuristes, Lausanne, L'Âge d'H omme, 1975.

Canosa, 1991: M ichele Canosa, «Il crepuscolo dei divi (muti e italiani)», Cinegrafie, vol. 2, no 4, 1991, p. 123-124.

Capek, 1924: Karel Čapek, «R.U.R. Comédie utopiste en trois actes et un prologue», trad. H. Jelinek, LesC ahiers dramatiques, vol. 2, n 21, 1924.

G eser, 1996 : Guntram Geser, Fritz Lang. M etropolis und D ie Frau im M ond, M eitingen, Lorian-Verlag H einrich Wimmer, 1996.

H auser, 1962: Arnold $\mathrm{H}$ auser, The Social $\mathrm{H}$ istory of Art, Vol. $4: \mathrm{N}$ aturalism, Impressionism, the Film Age, London, Routledge \& Kegan Paul, 1962.

Léger, 1922: Fernand Léger, «Essai sur la valeur plastique du film d'Abel Gance La Roue», Comœdia, 16 décembre 1922.

Léger, 1926: Fernand Léger, «A N ew Realism - The O bject (Its Plastic and Cinematographic Value) », The Little Review, 1926, p. 7. 
Lista, 1973: Giovanni Lista, Futurisme. M anifestes, proclamations, documents, Lausanne, L'Âge d'H omme, 1973.

Marinetti, 1910: Filippo Tommaso M arinetti, M afarka le futuriste. Roman africain, Paris, Sansot, 1910.

Martinelli, 1996: Vittorio M artinelli, II cinema muto italiano. I film degli anni venti. 1921, Torino, N uova Eri/Edizioni Rai, 1996.

Pirandello, 1925 : Luigi Pirandello, On tourne. Notes de Serafino Gubbio opérateur, Paris, É ditions du Sagittaire, 1925.

Rolland, 1947: Romain Rolland, La Révolte des machines ou La Pensée déchaînée, Paris, Pierre Vorms, 1947.

Tessari, 1973: Roberto Tessari, II mito della machina. Letteratura eindustria nel primo N ovecento italiano, M ilano, M ursia, 1973.

Vertov, 1972 : D ziga Vertov, Articles, journaux, projets, Paris, U .G.E, 1972.

Le mécanoïde et I'androïde: deux faces du mythe futuriste dans le cinéma d'avant-garde... 